\title{
Molecular Design of Novel Conjugated Polymers for Blue-Light-Emitting Devices
}

\author{
Sung Y. Hong \\ Department of Chemistrv, Institute of Natural Science, Kosin Chiversin; Pusan 606-701, Korea \\ Received March +, 2003
}

\begin{abstract}
A quantum-chemical study of conformations and electronic structures of polyheterocyclic derivatives with vinylenediheteroatom substituents at the 3- and 4-positions was performed to search for novel blue-lightemitting conjugated polymers. Conformational potential energy curves of the polymers were constructed as a function of the helical angle (a) through semiempirical Hartree-Fock band calculations at the Austin model 1 level. It is found that poly (3.4-vinylenedioxythiophene) possesses a quite flat curve in the range of $\alpha=51.4^{\circ}$. $120^{\circ}$. Replacing $\mathrm{S}$ atoms for $\mathrm{O}$ atoms greatly increases repulsion between the neighboring units, and thereby the units become perpendicular to one another. Because of the hydrogen bonding between $\mathrm{O}$ and $\mathrm{NH}$. poly (3.4vinylenedioxypyrrole) is predicted to be anti-coplanar and poly (3.4-vinylenediaminofuran) to be nearly anticoplanar. According to the modified extended Hückel band calculations. the HOMO-LUMO gaps (HLGs) of the polymers. unless the poly mer chains are twisted, are close to or slightly smaller than those of their respective mother polymers. Among the polymers, poly (3.4-vinylenediosythiophene) is presumed to be the most probable candidate for a blue-light emitter because its HLG is within the range of the electronic requirement for blue-light emitters
\end{abstract}

Key Words : Quantum-chemical investigation. Blue-light-emitting polymer. Conformation. Electronic structure. Heterocyclic polymers

\section{Introduction}

During the last decade. conjugated polymers for lightemitting diodes (LEDs) have attracted enormous attention because of their potential advantages (such as low operating voltages. low manufacturing cost and readiness for largearea displays) over inorganic materials. ${ }^{1}$ For red LEDs. a variety of poly'(3-alkylthiophene)s (P3ATs) with different length of alkyl groups have been introduced. ${ }^{2}$ But they have quite low fluorescence quantum yields. Poly'(p-phenyienevinylene)s (PPVs) with electron-withdrawing substituents also exhibit red-light emission. ${ }^{3}$ Green-light emission requires a larger band gap of material than red-light emission. Therefore. PPV and its derivatives are suitable for the green LEDs. PPV is the first polymeric LED material. whose emission peaks appear at $551 \mathrm{~nm}$ and $520 \mathrm{~nm}{ }^{+}$ Poly [2.(2'-ethy lhexyl)-5-butyl-1.4-phenylenevinylene] ${ }^{5}$ was reported to exhibit electroluminescence (EL) peak at $520 \mathrm{~nm}$ with a quantum efficiency of $3.2 \%$ for double layer devices. P3ATs with bulky substituents ${ }^{6}$ and head-to-head P3ATs ${ }^{7}$ possess sterically distorted conjugated backbones. resulting in green emission. Blue-light emission requires relatively large HOMO-LUMO gaps (HLGs) of materials. whereas most of conjugated polymers exhibit lower band gaps. Therefore. earlier studies on blue LEDs were done mainly on phenylene-based polymers. ${ }^{8.5}$ The quantum efficiency for a poly' $(p$-phenylene) device was reported to be only $0.01-$ $0.05 \%{ }^{8}$ Poly (9.9-dihexy fluorene) was demonstrated to enit light with a peak at $470 \mathrm{~nm}$ (corresponding to photon energy of $2.6 \mathrm{eV}$ ) ${ }^{9}$ Poly'(2-decyloxy-1.4-phenylene) exhibited an EL peak at $420 \mathrm{~nm}$ with a quantum efficiency of up to $3 \%$ for double-layer devices. ${ }^{10}$

Blue-light emitters have also been obtained through controlling conjugation length along conjugated backbones in several ways. "Imposing steric hindrance through bulky side groups is one way to tune color by distorting a conjugation chain ${ }^{67.12}$ This approach, however. gives rise to bringing emission from the more conjugated segments. Another way is to incorporate nonconjugated segments such as silylene. methylene and ether groups into a conjugated backbone. ${ }^{13}$ Broken conjugation has been also successfully achieved using $m$-phenylenes as an internupting block. ${ }^{i+}$ Moreover. insertion of $m$-phenylene units has demonstrated great enhancement of photoluminescence (PL) efficiency of the polymers. ${ }^{15}$ Our recent theoretical study ${ }^{16}$ has revealed that weak conjugation along $m$-phenylene-linked conjugated backbone results from the inherent nodal nature of the frontier molecular orbitals of the $m$-phenylene unit.

Although plenty of light-emitting polymers have been introduced. new efficient light-emitting polymers are still required for LEDs of commerce. In this work. we investigated conformations and electronic structures of polyheterocycles with vinylenediheteroatomic substituents at both 3- and 4positions to look for novel blue-light emitting polymers: poly (3.4-viny lenedithiothiophene) (PVDTT). poly'(3.4-vinylenedixoythiophene) (PVDOT), poly'(3.4-vinylenedioxypyrrole). (PVDOP), and poly (3.+-vinylenediaminofuran) (PVDAF) (see 1). For comparison. their mother polymers. ie.. polythiophene ( $\mathrm{PT}$ ), polypyrrole ( $\mathrm{PPy}$ ), and polyfuran ( $\mathrm{PFu}$ were included in the study: Among the polyheterocycles. PTs have been investigated extensively for light-emitters because of their environmental stability. thermal stability. 


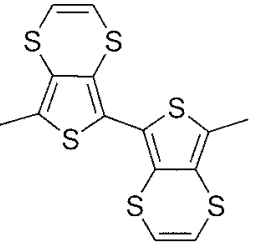

Poly (3,4-vinylenedithiothiophene) (PVDTT)

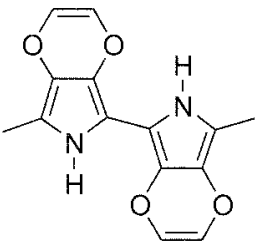

Poly (3,4-vinylenedioxypyrrole) (PVDOP)

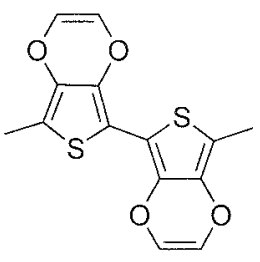

Poly(3,4-vinylenedioxythiophene) (PVDOT)

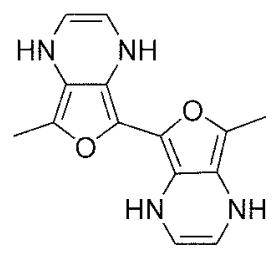

Poly(3,4-vinylenediaminofuran) (PVDAF) processability: mechanical strength and easy functionalization. Substitution at both 3- and 4-positions of PT induces conformational change toward distorted backbones and leads to emitting blue light. ${ }^{67.12} \mathrm{PPy}$ and PFu have been very rarely used as light emitters although they possess appropriate band gaps for blue-light emitters. Polyimide films containing furan were reported to exhibit intense blue PL with double peaks at 419 and $436 \mathrm{~nm}^{17}$

\section{Results and Discussion}

Methodology. Recent rapid growth of computer technologies and development of quantum-chemical program packages make it possible to calculate precisely molecular properties at a very high level. However. such a high level calculation (including electron correlation with a large basis set) for a polymeric system is still restricted to a small unit system in practice. ${ }^{18}$ For relatively large systems. ${ }^{19-23}$ polymeric structures have been obtained from the calculations on oligomeric structures or from the experimental measurements. Moreover it is well known that Hartree-Fock level calculations greatly overestimate the HOMO-LUMO gap of a conjugated polymer. And inclusion of electron correlation. on the other hand. leads to a significant underestimation of the gap. ${ }^{19}$ Therefore. one should perform numerical experiments to estimate how much electron correlation should be included to reproduce the experimentally-observed HOMOLUMO gap. ${ }^{-1}$ In this regard. well-defined semi-empirical methods are still attractive for a large or an unknown polymeric system.

We employed the solid-state version of the MNDO method (MOSOL) $)^{-4}$ with the AM1 hamiltonian to optimize geometrical parameters and to investigate the conformational behavior of the polymers. This version adopts the Born-von Kármán periodic boundary condition and Bloch functions
Table 1. Valence Shell Atomic Parameters Used in the Modified Extended Hückel Band Calculations

\begin{tabular}{ccccccccc}
\hline atom & $n$ & $l$ & $H_{i i}(\mathrm{eV})$ & $\zeta$ (a.ul.) & $n$ & $l$ & $H_{i i}(\mathrm{eV})$ & $\zeta($ a. ul. $)$ \\
\hline $\mathrm{H}$ & 1 & 0 & -13.60 & 1.300 & & & & \\
$\mathrm{C}$ & 2 & 0 & -21.40 & 1.625 & 2 & 1 & -11.40 & 1.625 \\
$\mathrm{~N}$ & 2 & 0 & -26.00 & 1.950 & 2 & 1 & -13.40 & 1.950 \\
$\mathrm{O}$ & 2 & 0 & -32.30 & 1.975 & 2 & 1 & -14.80 & 1.975 \\
$\mathrm{~S}$ & 3 & 0 & -20.00 & 2.117 & 3 & 1 & -13.30 & 2.117 \\
\hline
\end{tabular}

for crystal calculations. The AM1 method has been successfully used to investigate geometrical structures and conformations of large molecules in particular. although the method yields low rotational barriers. ${ }^{25}$ It also has produced excellent agreement with the experimental observation in the case of polymers. ${ }^{16}$ For the geometrical optimizations. we chose 6 wave vectors with a regular interval from 0 to $\pi / a$ (where $a$ is a translation vector), and imposed the $C_{t r}$ constraint on the monomer units.

When the neighboring local rotational axes of a polymer chain are not collinear, a helical conformation is more stable than an alternately twisted structure. ${ }^{16}$ Therefore. we examined conformational behavior of the polymers by rotating the rings consecutively in the same direction. producing a helical structure. Because a helical symmetry operator is not included in our MOSOL version. we determined a helical angle $(\alpha)$ of the chains by the number $(n)$ of the monomer units in the repeat unit. with the relationship $\alpha=$ $\left(m \cdot 360^{\circ}\right) / n$ of where $m$ is any integer. representing the number of turns which the unit cell makes. At each helical angle. a torsion angle between the adjacent rings is optimized with other geometrical parameters. The repeat unit cells contain up to 12 monomer units for PT. PPy and $\mathrm{PFu}$. and up to 8 monomer units for the other polymers.

Electronic properties were calculated by using the AMl optimized structures in the modified extended Hückel (MEH) method. ${ }^{26}$ This method expresses the off-diagonal elements of the EH method in a new form. which has an additional distance-dependent empirical factor. This was parameterized to reproduce a $\lambda_{\max }$ value for the $\pi-\pi^{*}$ transition of a conjugated polymer. not an onset value that has been usually referred to a band gap. This approach has predicted $\lambda_{\text {max }}$ values of a variety of conjugated polymers with fairly good accuracy: compared to experimental values. $2 x-29$ Atomic parameters used in the MEH calculations are presented in Table 1.

Polythiophene Systems. As shown in Figure 1. it is predicted that PT would possess two stable conformations: anti-coplanar and 12/1 helix. Refer to Table 2 for torsion angles of the polymers under investigation at each helical angle. In the $12 / 1$ helix. the $\mathrm{H} \cdots \mathrm{H}$ distance is calculated to be $2.39 \mathrm{~A}$. which is double the van der Waals radius $(1.2 \mathrm{~A})$ of $\mathrm{H}$ atom. The $\mathrm{S} \cdots \mathrm{H}$ distance in the anti-coplanar structure is estimated to be $2.93 \mathrm{~A}$. close to the sum $(3.0 \mathrm{~A})$ of van der Waals radii of $\mathrm{S}$ and $\mathrm{H}$ atoms. The two conformations are nearly isoenergetic and separated by an energy barrier of $0.8 \mathrm{kcal} / \mathrm{mol}$ per monomer unit. The potential well centered 


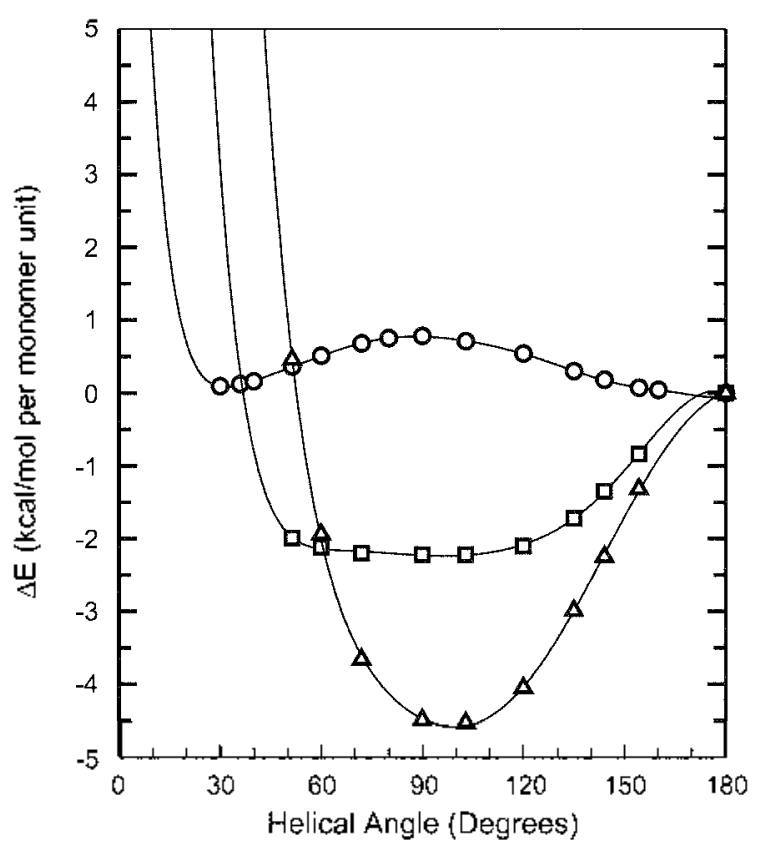

Figure 1. Potential energy curves of PT $(--)$, PVDOT $(\sqsupset)$, and PVDTT $(\therefore)$ as a function of the helical angle of the conjugated backbones.

at $\alpha=180^{\circ}$ is much broader than the well centered at $\alpha=30^{\circ}$. A similar potential energy' curve for PT has been produced through extended Hückel theory (EHT) calcu-

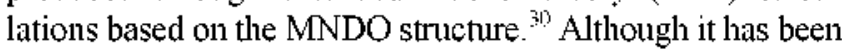
generally accepted that PT is of anti-coplanar conformation ${ }^{31}$ and that all non-substituted oligothiophenes are quasiplanar. ${ }^{32}$ scanning tunneling microscopic experiments have revealed the coexistence of the $\mathrm{sm}$ - and anti-conformations in PT and alkyl-substituted PTs ${ }^{\dot{j}}$

The relative populations of the minima depend on their statistical weights. which include contributions from both the potential energy and the entropy. ${ }^{3.4}$ If two potential wells have similar depths the broader potential well has a larger statistical weight due to the proportionately larger contribution from the conformational entropy because more conformational microstates are available. ${ }^{35}$ Therefore. dominant abundance of the anti-coplanar conformation of PT is not only due to the solid-state packing but due to the entropic contribution. The HLG of anti-coplanar PT is estimated to be $2.32 \mathrm{eV}$. in good agreement with the experimental $\lambda_{\text {max }}$ values $(2.5-2.7 \mathrm{eV}){ }^{36}$ Interestingly, the HLG for

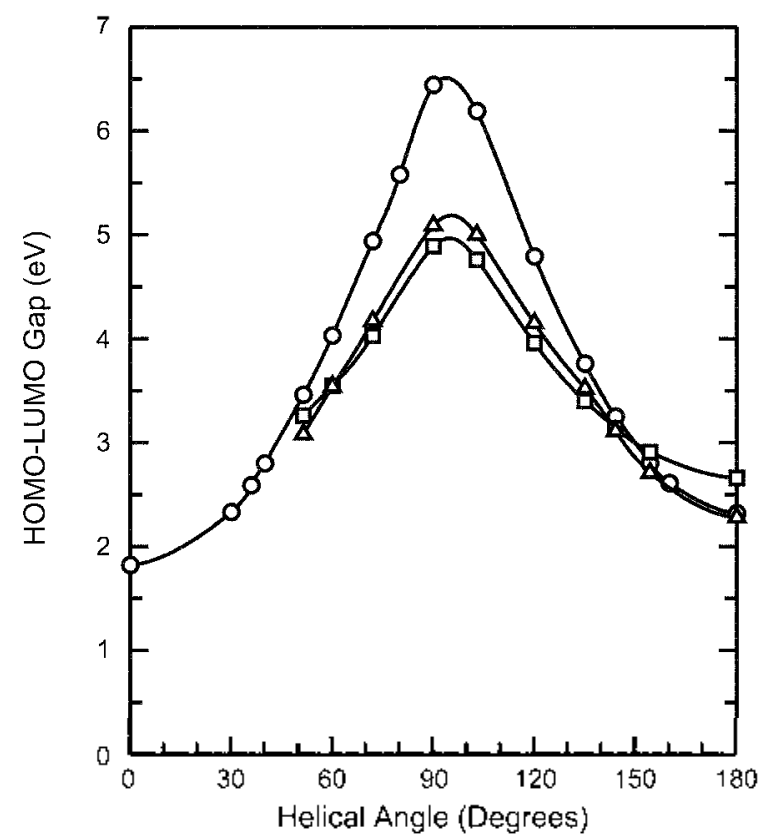

Figure 2. HOMO-LUMO gaps of PT ( $\square)$, PVDOT ( $\sqsubset$ ), and PVDTT $(\therefore)$ as a function of the helical angle of the conjugated backbones.

the helical PT with $\alpha=30^{\circ}$ is estimated to be accidentally the same as that for the anti-coplanar structure (see Figure 2). This fact would prevent UV-Vis spectroscopic investigations from distinguishing two conformations.

Substituting vinylenedithio groups at the 3- and 4positions greatly increases repulsive interaction between adjacent monomer units. making the monomer units perpendicular to one another. In fact. the potential energy curve of PVDTT exhibits a deep minimum at a helical angle between $\alpha=90^{\circ}$ and $102.9^{\circ}\left(\tau=84.8^{\circ}-98.6^{\circ}\right)$. The closest $\mathrm{S}$ $\cdots \mathrm{S}$ distance is $2.93 \mathrm{~A}$ in the anti-coplanar structure. which is quite small compared to double the $\mathrm{S}$ van der Waals radius $(1.80 \mathrm{~A}){ }^{37}$ and $3.85 \mathrm{~A}$ in the $7 / 2$ helical structure $(\alpha=$ $\left.102.9^{\circ}\right)$. The anti-coplanar conformation is higher in energy than the $7 / 2$ helical conformation by $4.5 \mathrm{kcal} / \mathrm{mol}$ per monomer unit. The HLGs of PVDTT are $5 \mathrm{eV}$ and $2.3 \mathrm{eV}$ in the $7 / 2$ helical conformation and in the anti-coplanar structure. respectively. It is found that introduction of the vinylenedithio group perturbs both frontier molecular orbitals of PT backbone. raising the MO energy levels about the same amount (by $0.7 \mathrm{eV}$ ). As a result the HLG of

Table 2. Torsion Angles of the Polymers at Each Helical Angle under Investigation (Angles in Degrees)

\begin{tabular}{lcccccccccccccccc}
\hline $\begin{array}{l}\text { Helical } \\
\text { Angle }\end{array}$ & 0.0 & 30.0 & 36.0 & 40.0 & 51.4 & 60.0 & 72.0 & 80.0 & 90.0 & 102.9 & 120.0 & 135.0 & 144.0 & 154.3 & 160.0 & 180.0 \\
\hline PT & 0.0 & 11.3 & 22.4 & 28.4 & 42.7 & 52.6 & 66.0 & 74.7 & 85.6 & 99.3 & 117.6 & 133.2 & 142.6 & 153.3 & 159.3 & 180.0 \\
PPy & 0.0 & 6.4 & 10.0 & 14.5 & 32.4 & 43.9 & 59.2 & 69.1 & 81.1 & 96.0 & 115.4 & 131.6 & 141.4 & 152.4 & 158.5 & 180.0 \\
PFu & 0.0 & 3.8 & 5.7 & 7.3 & 15.2 & 29.4 & 50.3 & 62.2 & 75.6 & 91.8 & 112.6 & 129.7 & 140.1 & 151.5 & 157.8 & 180.0 \\
PVDTT & 0.0 & - & - & - & 37.0 & 49.3 & 64.4 & - & 84.8 & 98.6 & 116.2 & 132.3 & 141.0 & 151.4 & - & 180.0 \\
PVDOT & 0.0 & - & - & - & 40.7 & 51.3 & 65.2 & - & 85.1 & 98.9 & 116.8 & 132.7 & 142.6 & 152.7 & - & 180.0 \\
PVDOP & 0.0 & - & - & - & 30.1 & 42.3 & 58.3 & - & 80.7 & 95.7 & 115.8 & 132.1 & 142.3 & 152.9 & - & 180.0 \\
PVDAF & 0.0 & - & - & - & 31.4 & 36.8 & 52.1 & - & 76.2 & 92.2 & 113.3 & 130.2 & 140.7 & 151.7 & - & 180.0 \\
\hline
\end{tabular}



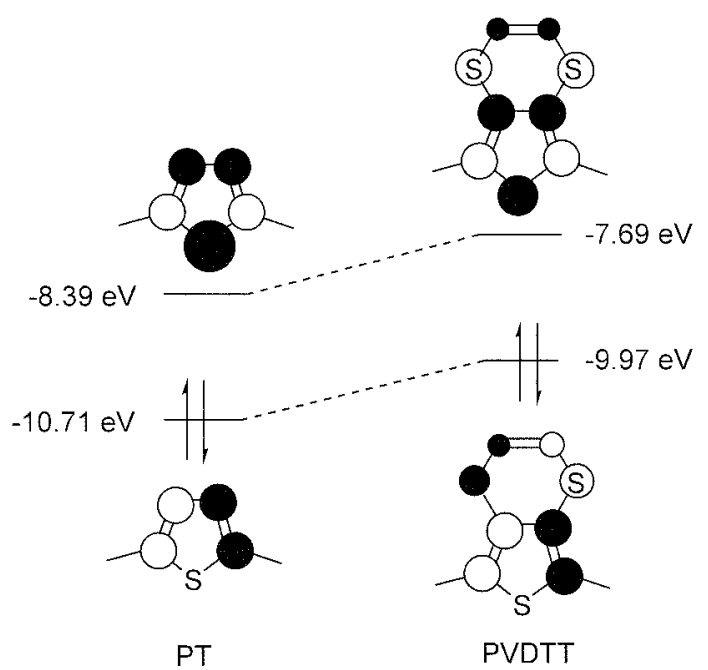

Figure 3. Frontier molecular orbital correlation between polythiophene and poly( $3,4-$-vinylenedithiothiophene) in the anti-coplanar confomation.

PVDTT in the anti-coplanar conformation is similar in value to that of PT. $\pi$-Type frontier molecular orbital interactions of the group with the PT backbone are illustrated in Figure 3.

Replacing $\mathrm{O}$ atoms for $\mathrm{S}$ atoms in the vinylenedithio groups reduces the repulsive interaction between neighboring monomer units. This leads to the quite flat potential energy curve of PVDOT at the helical angles in the range of $\alpha=51.4^{\circ}-120^{\circ}$ (see Figure 1). Therefore, we expect a variety of helical structures (3-7 monomer units per turn) for PVDOT. At $\alpha=51.4^{\circ}\left(\tau=40.7^{\circ}\right)$. where the repeat unit consists of 7 monomer units ( $7 / 1$ helix), the closest $\mathrm{O} \cdots \mathrm{O}$ distance is $2.9 \mathrm{~A}$. which is close to double the van der Waals radius ( $1.5 \mathrm{~A}$ ) for $\mathrm{O}$ atom. At $\alpha=120^{\circ}\left(\tau=116.8^{\circ}\right)$, where a $3 / 1$ helical structure is formed. the $\mathrm{S} \cdots \mathrm{O}$ distance is $3.54 \mathrm{~A}$. slightly larger than the sum of the van der Waals radii of $\mathrm{S}$ and $O$ atoms. In this conformational region. HLG of PVDOT extends from $3.3\left(\alpha=51.4^{\circ}\right)$ to $4.9 \mathrm{eV}\left(\alpha=90^{\circ}\right)$. Therefore. we expect a very broad emission peak of PVDOT near the blue region. The orbital interactions near the Fermi level are similar to those found in PVDTT. In the anti-coplanar structure the HOMO and LUMO energy levels are raised by $0.93 \mathrm{eV}$ and $1.26 \mathrm{eV}$ respectively. compared to those of PT.

Polypyrrole Systems. Figure 4 shows potential energy curves of PPy and PVDOP. The conformational curve for PPy exhibits two local minima at $\alpha=51.4^{\circ}$ and $180^{\circ}$. similar to that for PT. The former corresponds to the $7 / 1$ helical structure with $\tau=32.4^{\circ}$. The potential well centered at $\alpha=180^{\circ}$ is much broader than the well centered at $\alpha=51.4^{\circ}$. The anti-coplanar structure is more stable than the $7 / 1$ helical structure by $0.9 \mathrm{kcal} / \mathrm{mol}$ per monomer unit. The height of the rotational energy barrier from the anti-coplanar conformation to the $7 / 1$ helical conformation is estimated to be $1.4 \mathrm{kcal} / \mathrm{mol}$ per monomer unit. Earlier EHT calculations for PPy ${ }^{30}$ produced a similar conformational potential curve. but large stability of the anti-coplanar conformation and a large energy barrier compared to our result. Unlike the case of PT, the two conformers exhibits different HLGs: $3.78 \mathrm{eV}$

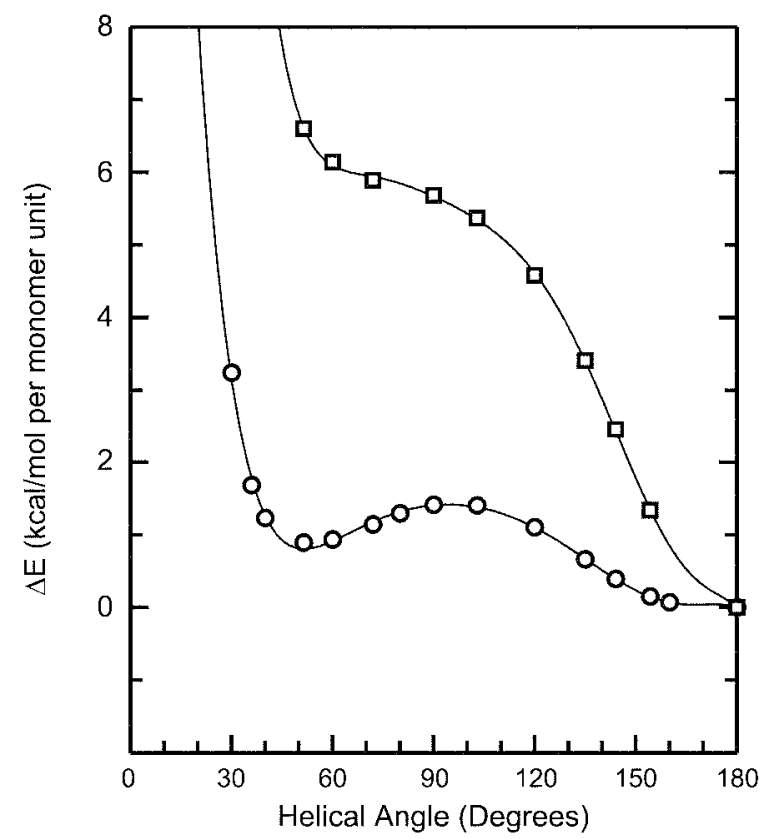

Figure 4. Potential energy curves of PPy ( $a$ ) and PVDOP ( $\sqsubset$ ) as a function of the helical angle of the conjugated backbones.

at $\alpha=51.4^{\circ}$ and $3.15 \mathrm{eV}$ at $\alpha=180^{\circ}$ (see Figure 5). The predicted HLG of the anti-coplanar structure is in excellent agreement with the experiment UV-Vis $\lambda_{\max }$ values $(3.2$ eV). ${ }^{38}$

Only one minimum is located in the potential curve for PVDOP. This is because. in PVDOP, $\mathrm{NH}^{\cdots} \mathrm{O}$ hydrogen bonds are formed between two adjacent monomer unit. greatly stabilizing the anti-coplanar structure. The distance between the $\mathrm{NH}$ and $\mathrm{O}$ atoms is $2.47 \mathrm{~A}$. which is much smaller than the sum $(2.7 \mathrm{~A})$ of van der Waals radii of $\mathrm{H}$ and

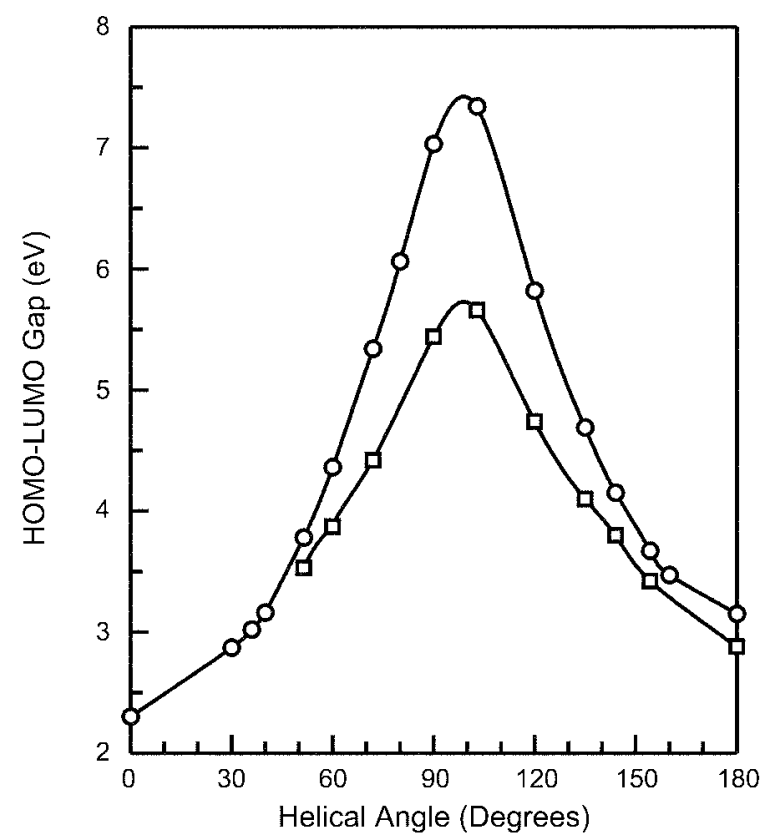

Figure 5. HOMO-LUMO gaps of PPy ( $~-$ ) and PVDOP ( $\sqsupset$ ) as a function of the helical angle of the conjugated backbones. 


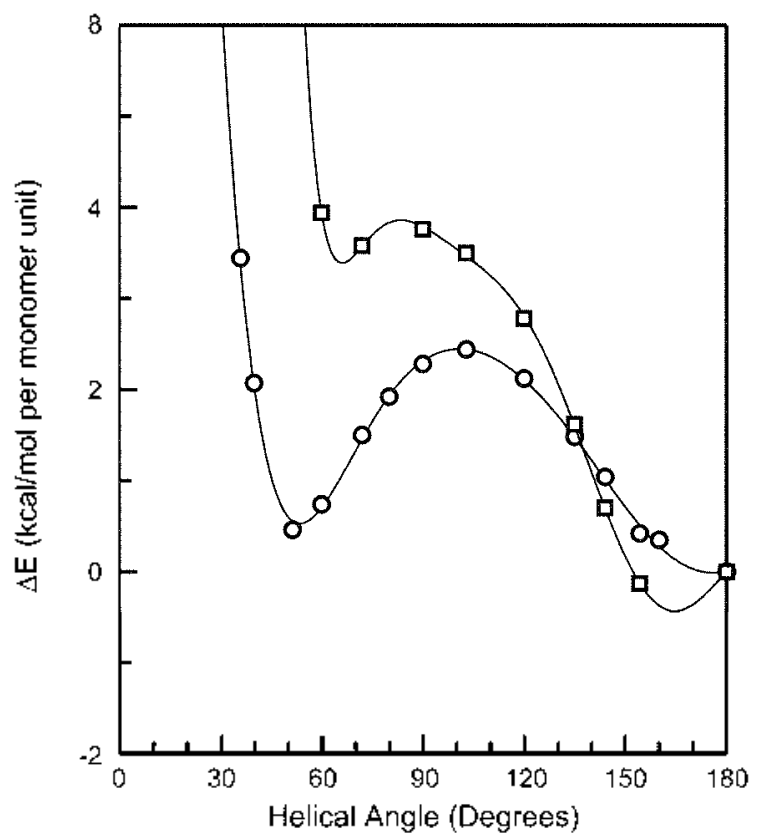

Figure 6. Potential energy curves of PFu ( ) and PVDAF ( $\sqsupset$ ) as a function of the helical angle of the conjugated backbones.

$\mathrm{O}$ atoms. The HLG of anti-coplanar PVDOP is estimated to be $2.92 \mathrm{eV}$. close to that of anti-coplanar PPy: but not large enough for a blue-light emitter. The orbital interactions of the vinylenedioxy group with PPy backbone are similar to those found in PVDTT, raising HOMO by $0.98 \mathrm{eV}$ and LUMO by $0.75 \mathrm{eV}$.

Polyfuran Systems. As in the case of PT and PPy, two minima are found in the conformational potential energy curve for PFu. corresponding to the $7 / 1$ helix $\left(\alpha=51.4^{\circ}\right.$ with $\tau=15.2^{\circ}$ ) and anti-coplanar structure (see Figure 6). The former is slightly ligher in energy than the latter by $0.46 \mathrm{kcal} / \mathrm{mol}$ per monomer unit. The two conformations are separated with a rotational energy barrier of $2.44 \mathrm{kcal} / \mathrm{mol}$ per monomer unit. In the $7 / 1$ helical structure. the closest $\mathrm{O} \cdots \mathrm{O}$ distance is calculated to be $2.77 \mathrm{~A}$, which is slightly smaller than the double the van der Waals radius of $O$ atom. The anti-coplanar stnucture is predicted to possess the HLG of $3.07 \mathrm{eV}$ in excellent agreement with an experimental $\lambda_{\text {max }}$ value $(3.0 \mathrm{eV}){ }^{39}$ The $7 / 1$ helical structure is predicted to possess a large $\mathrm{HLG}$ of $3.78 \mathrm{eV}$.

PVDAF shows two local minima near $\alpha=72^{\circ}$ and $160^{\circ}$. The rod-like structure is more stable than the coil-like structure by $c$ ar $3.7 \mathrm{kcal} / \mathrm{mol}$ per monomer unit. The large stabilization of the rod-like structure is due to the $\mathrm{NH} \cdots \mathrm{O}$ hydrogen bonding between neighboring unit. The hydrogen bonding is however not strong enough to keep the chain completely planar. Such weak hỵdrogen bonding in furan system has been ascribed to the delocalized oxygens lone pair electrons in the $\pi$ system of the ring. ${ }^{\text {th }}$ The HLGs of PVDAF are predicted to be $3.2 \mathrm{eV}$ at $\alpha=154.3^{\circ}$ and $2.91 \mathrm{eV}$ at $\alpha=180^{\circ}$. The HLG of the anti-coplanar structure is slightly smaller than that of $\mathrm{PFu}$. and not large enough for a blue-light emitter. Perturbation due to the vinylenediamino group is similar to that found in PVDTT. The HOMO and

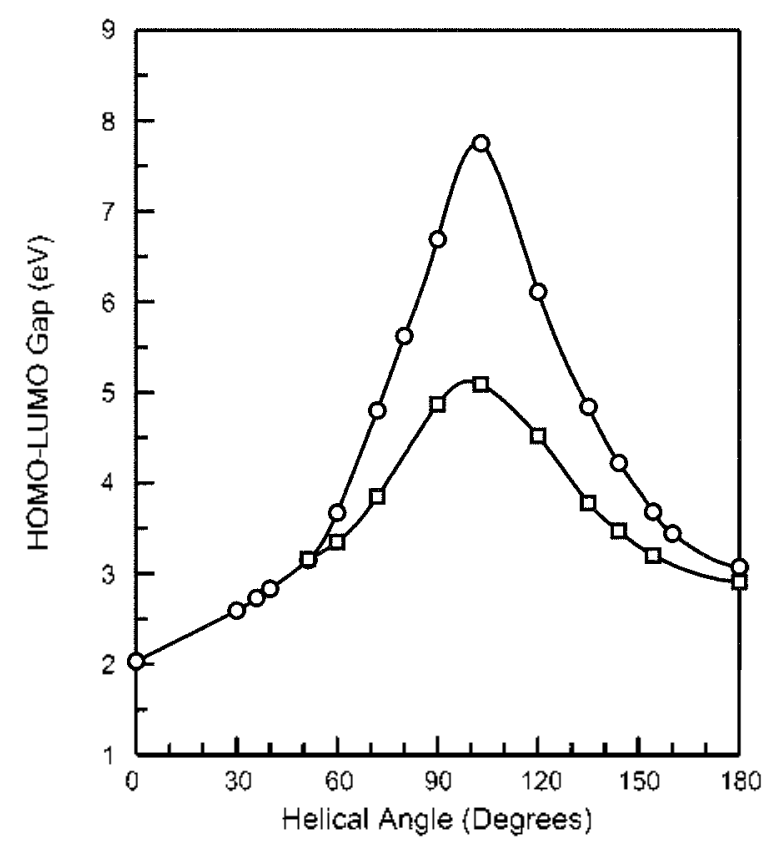

Figure 7. HOMO-LUMO gaps of PFu ( $\square$ ) and PVDAF ( $\sqsubset$ ) as a function of the helical angle of the conjugated backhones.

LUMO energy levels of PVDAF are higher than those of $\mathrm{PFu}$ by 1.38 and $1.22 \mathrm{eV}$. respectively.

\section{Conclusions}

It is found that substituting vinylenediheteroatomic groups at the 3-and 4-positions of heterocyclic polymers raises the HOMO and LUMO energy levels about the same amounts. As a result. unless the polymer chains are twisted. the substitution does not significantly change HLG values of the polymers. compared to those of their mother polymers. Owing to the hydrogen bonding between $\mathrm{O}$ and $\mathrm{NH}$. PVDOP is predicted to be anti-coplanar and PVDAF to be quasi-planar. Therefore. their HLGs are close to those of PPy and $\mathrm{PFu}$. respectively. and slightly lower than the electronic requirement for blue-light enitters.

On the other hand. PVDTT is twisted with neighboring units perpendicular to one another in order to avoid the strong $S \cdots S$ repulsion between the neighboring rings. possessing a too large HLG for a blue-light enitting polymer. However. the possibility of PVDTT in the solid state to emit blue-light cannot be completely ruled out if packing in the solid state increases the planarity of the polymer chain so that the HLG drops to $3.5 \mathrm{eV}$ or so. In PVDOT, such a strong repulsion is more or less relieved and a quite flat potential energy curve is found in the range of $\alpha=51.4^{\prime}-120^{\prime \prime}$. In this conformational range the HLG of the polymer extends from 3.2 to $4.9 \mathrm{eV}$. In conclusion, among the polymers we have investigated. PVDOT is expected to be the most probable candidate for a blue-light emitter.

Acknowledgement. The author wishes to acknowledge the financial support of the Korea Research Foundation made in the program year of 1998 (1998-015-D00152). 


\section{References}

1. For example. see one of the review papers: Kraft. A.; Grimsdale, A. C.: Holnes. A. B. Angew: Chem. Int Ed 1998, 37.402

2. Chen. F.: Mehta. P. G.: Takiti. L.: McCullough. R. D. J. Mater. Chem. 1996. 6. 1763. Ohmori. Y: Uchida. M.: Muro. K: Yoshino. K. Ipn. J. Appt. Phns. 1991. 30. L1938. Braun. D.: Gustatsson. G.: MoBranch, D: Heeger, A. I. J. Appt. Phns, 1992, 72. 564. Greenham, N. C.: Brown, A. R.: Bradley, D. D. R. Friend, R. H. Symh. Wet 1993.57.4134.

3. Greenham. N. C.: Friend. R. H.: Bradley. D. D. R. Ad. Mater. 1994. 6.491. Greenham. N. C.: Moratti. S. C.: Bradley. D. D. R: Friend. R. H.: Holms. A. B. Nature 1993. 365. 628. Burroughes. J. H.: Bradlev. D. D. C.: Brown A. R: Marks. R. N.: Mackav. K.: Friend, R. H.: Burns, P. L.: Holmes, A. B. Nature $1990,347,539$. Gurge. R. M.: Sarker. A.: Lahti. P. M: Hu. B.: Karasz, F. E. Hacromolectles 1996. 29. 4287

4. Burroughes. J. H.: Bradley. D. D. C.: Brown. A. R.: Marks. R. N.: Mackay. K.: Friend. R. H.: Burns. P. L.: Holmes. A. B. Nature $1990,347,539$

5. Andersson. M. R.: Yll. G.: Heeger. A. J. Sinth. Met. 1997. 85. 1275 .

6. Berggren. M: Gustatsson. G.: Inganäs. O: Andersson. M. R.: Wennerström. O.: Hjertberg. T. Adt: Mater 1994. 6. 488. Berggren. $\mathrm{M}$ : Inganäs. $\mathrm{O}$ : Gustatsson. G.: Rasmusson. J.: Andersson, M. R: Hjertberg. T: Wennerström, O. Nature 1994. 372.444

7. Gill, R. E.: Malliaras, G. G.: Wildeman. J: Hadziioannou. G. Ad: Mater: 1994, 6. 132.

8. Grem. G.: Leditzhy. G.: Ulrich. B.: Leising. G. Ad: Mater. 1992. 4. 36. Grem. G.: Leditzhy. G.: Ulrich. B.: Leising. G. Stmth. Met. $1992,51,383$.

9. Olmnori, Y: Uchida, M. Muro. K.: Yoshino, K. Jpn. J. Appl. Phns. 1991, 30. L1941

10. Yang. Y.: Pei. Q.: Heeger. A. J. J.Appl. Phys. 1996, 79.934.

11. For a recent review paper on blue-light-emitting polymers, see Kim. D. Y.: Cho. H. N.: Kim. C. Y. Prog. Potwm. Sai 2000.25. 1089 .

12. Andersson. M. R: Berggren. M.: Inganäs, O: Gustafsson. G.: Gustafsson-Carlberg. J. C.: Selse, D.: Hjertberg. T: Wennerström. O. Macromolectles 1995, 28.7525. Miyazaki. Y: Yamamoto. T. Chem. Lett. 1994, 41 .

13. Miliaras. G. G.: Herrema. J. K.: Wildeman. J.: Wieringa. R. H.: Gill, R. E.: Lampoura. S. S.: Hadzioannou, G. Adt: Hater 1993 5.721. Yang. Z.: Karasz. F. E.: Geise. H. I. A facronolecules 1993. 26, 6570. Zvung. T.: Hwang. D.-H.: Kang. I.-N.: Shim, H.-K.: Hwang. W.Y.: Kim. J.-J. Chem. Mater. 1995. 7. 1499. Ryu. M. K: Lee. J.H.: Lee, S.-M.: Kim. H.-K.: Zvung. T. Pohm. Mater. Sic. Eng. 1996. 75. 408. Kim. Y.: Kwon. S.: Yoo. D.: Rubner. M. F: Wrighton. M. S. Chent Mater: 1997, 9, 2699. Gowri, R: Mandal D.: Shivkumar, B.: Ramakrishnan. S. Macromolecules $1998,31,1819$

14. Musfeldt. J. L.: Reynolds. J. R.: Tanner. D. B.: Ruiz. J. P.: Wang. J.: Pomerzntz. M. J. Pohm. Sci. Part B: Pohm. Plys. 1994, 32. 2395. Kang. B. S.: Seo. M.-L.: Jun. Y. S.: Lee. C. K.: Shin. S. C. Chem. Conmm. 1996, 1167. Kang. B. S. Kinn. D. H. Lim. S. M.: Kim, J.: Seo, M.-L.: Bark, K.-M.: Shin. S. C. Hacromolecules $1997,30,7196$
15. Cho. H. N: Kim, D. Y: Kim, Y. C.: Lee. J. Y: Kim, C. Y. Adt: 1/ater: 1997, 9, 326. Pang. Y: Li. J: Hu. B.; Karasz. F. E. Macromolecules 1998.31 .6730 .

16. Hong. S. Y: Kim. D. Y: Kim. C. Y: Hoffimann. R Macrontolecules 2001 . 34. 6474

17. Pyo. S. M.: Kim. S. L.: Shin, T. J.: Park. H. K.: Ree. M.; Park. K. H.: Kang. J. S. A facrontolectles 1998. $31,4777$.

18. Springborg. M Int. J. Quantum Chem 2000. 77,843.

19. Salzner. U.: Lagowski. I. B.: Pickup. P. G.: Poirier. R. A. d. Comput. Chem 1997.18. 1943

20. Salzner. U.: Pickup. P. G.: Poirier. R. A.: Lagowski. J. B. J. Phys. Chent A 1998. 102.2572.

21. Vaschetto, M. E; Retamal, B. A.; Monkman. A. P.: Springborg, M. J. Plys. Chem \& 1999, 103, 11096 . Lim. S. L.; Tan. K. L: Kang. E. T.: Chin. W. S. J. Chem. Phus. 2000.112. 10648.

22. Palmer. I. T.: Ladik. T. J. Comput Chem 1994. 15.814.

23. Champagne. B.: Mosley. D. H.: Fripiat. J. G.: Anré. T.-M. Phns. Rer: $B$ 1996-II. $5+.2381$

24. Dewar, M. J. S.: Zoebisch. E. G.: Healy, E. F: Stewart. J. J. P. J Am. Chem. Soc. 1985. 107. 3902 . Stewart, J. J. P. $Q C P E$ Bull. 1985. 5. 62. Cui. C. X.: Kertesz. M J. Am. Chem. Soc. 1989. 111. 4216.

25. Fabian. W. M. F. J. Comput Chem. 1988.9.369.

26. Hong. S. Y; Marynick, D. S, J. Chent. Phns. 1992,96. 5497

27. Hong. S. Y: Song. J. M. J. Chent. Phts 1997, J07, 10607

28. Hong. S. Y. Bull Kowan Chem. Soc. 1999. 20. 42.

29. See the following references to compare the MEH prediction and experimental measurement for polysilole: Yamagauchi. S.: Goto. T.: Tamao. K Angew Chent Int Ed 2000. 39. 1695. Hong. S. Y.: Kwon. S. J.: Kim, S. C. J. Chem. Phss 1996. 104, 1140 . Hong, S. Y.: Song. J. M. Sinh. Met. 1997, 85, 1113.

30. Cui. C. X.: Kertesz, M. Phus. Rev B 1989-I t0, 9661

31. Brückner. S.: Poizio. W. Mfakromol. Chem. 1988. 189. 961.

32. van Bolhuis. F.: Wytherg. H: Havinga. E. E.: Meijer. E. W. Staring. E. G. T. Swh. Met 1989. 30.381. Horowitz. G.: Bachet. B.: Yassar, A.; Lang, P.: Demanze. F: Fave. J. L.; Garnier, F. Chent. Mater: 1995. 7. 1337.

33. Caple. G.: Wheeler, B. L.: Swift, R.: Porter, T. L.; Jeffers. S. d. Phus. Chem. 1990. 94. 5639. Lacaze. E.: Uydal. K: Bodö. R: Garbarz. T.: Salaneck. W. R.: Schott. M. J. Polum. Sci. Polvm. Phos Ed 1993. 31. 111. Mena-osteritz. E.: Meyer. A.: LangeveldVoss, B. M. W. Janssen, R. A. J.; Meijer, E. W. Bäuerle. P. Angew Chem. Wht Ed. 2000. 39,2680

34. Scheraga, H. A. Chem Rev $1971,71,195$

35. Richards. W. G. J. Theor: Biol 1974. 43. 389.

36. Kaneto. K.: Yoshino. K.: Inuishi. Y. Solid State Commum 1983. 46. 389. Chung. T.-C.: Kasufmant. I. H.: Heeger. A. T.: Wudl. F Phis: Ren: B 1984. 30,702.

37. Values of van der Waals radii from Bondi. A. J. Phns. Chent 1964. 68. 441 .

38. Street. G. B.: Clarke. T. C.: Krounbi. M.: Kanazawa. K.: Lee. V: Pfluger. P.: Scott. J. C.: Weiser. G. Mol. Const. Liq. Cryst 1982. 83. 253. Bredas. T. L.: Scott. T. C.: Yakushi. K.: Street. G. B. Phws Rev: $B$ 1984, 30.1023.

39. Zotti, G.: Schiavon. G.: Comisso. N.: Berlin. A.; Pagani, G. Swmt. 1fat. $1990,36,337$

40. Nobeli. I.: Price. S. L.: Lommerse J. P. M.: Taylor. R. $J$. Comput Chent 1997. 18.2060. 